RYsZARda RempaŁa (Warszawa)

\title{
TWO HEDGING POINTS POLICY FOR AN UNRELIABLE MANUFACTURING SYSTEM
}

Abstract. This paper deals with an unreliable manufacturing system in which limited backlog is allowed. An admissible production policy is described by two decision parameters: upper and lower hedging points. The objective is to find the optimum hedging points so as to minimize the long run average expected cost under an additional condition. The condition expresses a constraint for the limiting probability of the event that the system stays at the lower hedging point, which corresponds to a limit of backlog. The cost consists of two parts: holding inventory cost and shortage cost. The optimum hedging points are determined.

1. Introduction. The paper deals with a version of unreliable manufacturing system with an average cost criterion discussed in [2] and [4]. In both papers the production policy was described by one decision parameter, the so-called hedging point. In the model considered here the production policy is described by two decision parameters: upper and lower hedging points. By means of such a policy we are able to consider a production system in which a limited backlog is allowed. In [2] the total inventory can be negative, which corresponds to unlimited backlog, while in [4] no backlog is allowed. So we discuss a model which in a sense combines both situations.

The model may be described in the following way. The system has two states: "up-state" and "down-state". If the system is in the up-state, it can produce continuously over time at a rate $u \in[0, r], r>0$. If the system is in the down-state it cannot produce. The time interval between failures is random and modelled by an exponentially distributed random variable with parameter $\lambda_{u}$, while the repair time is an exponentially distributed random variable with parameter $\lambda_{d}$. The demand rate, say $v$, is constant, so the

2000 Mathematics Subject Classification: Primary 90B05.

Key words and phrases: unreliable manufacturing system, average inventory cost, two hedging points. 
product is continuously depleted at the demand rate. If the demand cannot be satisfied it causes a shortage. So the corresponding inventory process can take positive as well as negative values. Both kinds of state are limited: the positive states are limited by an upper hedging point $z_{1}$ and negative ones by a lower hedging point $-z_{2}$. Both $z_{1}, z_{2}$ are treated as decision variables. The lower hedging point $-z_{2}$ makes the shortage of size up to $z_{2}$ allowed and backordered. The shortage of size over $z_{2}$ is lost forever. (Issues relating to production systems with limited backlog have attracted considerable attention in [5]-[7].) In the model discussed here positive inventories are assessed a cost at a rate of $c^{+}$dollars per unit commodity per unit time, while negative inventories are assessed a similar cost of $c^{-}$. The case that the demand during the stockout period is lost is stressed in a different way. There is an additional constraint for the limiting probability of the shortage of size over $z_{2}$. The constraint is discussed in Section 4 .

The paper is organized as follows. In Section 1 the mathematical description of the inventory process and the corresponding optimization problem are given. The limit distribution of the process is obtained in Section 3. Another formulation of the optimization problem is presented in Section 4 . The solutions of the problem for all cases considered are obtained in Sections 5 and 6 .

2. Inventory process. Mathematical description. The description of the model is similar to that given in [2] and [4]. Let $\xi_{n}, \eta_{n}$ be random variables describing the $n$th up-time and $n$th down-time of the system. We assume that $\left\{\xi_{n}\right\},\left\{\eta_{n}\right\}$ are two sequences of mutually independent random variables, $\xi_{n}$ are i.i.d., $\eta_{n}$ are i.i.d. and $P\left\{\xi_{n}<x\right\}=1-e^{-\lambda_{u} x}$, $P\left\{\eta_{n}<x\right\}=1-e^{-\lambda_{d} x}$ for $x \geq 0$. We specify the model more precisely in (a)-(d) below.

(a) Let

$$
I(t)= \begin{cases}1 & \text { if the system is up at time } t, \\ 0 & \text { if the system is down at time } t .\end{cases}
$$

(b) Let $z_{1} \geq 0, z_{2} \geq 0$ be the hedging parameters, $X_{z_{2}}(t)$ be the inventory level of the product at time $t$, and $u(t)$ be the production rate at time $t$. So

$$
u(t)= \begin{cases}0 & \text { if } I(t)=0 \\ \bar{u} \in[0, r] & \text { if } I(t)=1 .\end{cases}
$$

The process is modelled as follows:

where

$$
X_{z_{2}}(t)=\max \left(-z_{2}, Y(t)\right), \quad t \geq 0,
$$

$$
\frac{d}{d t} Y(t)=u(t)-v, \quad Y(0)=y_{0} \in\left[-z_{2}, z_{1}\right],
$$

and $v$ is a constant such that $0<v<r$. 
(c) Following [4] assume that an admissible production policy is of the form

$$
u_{z_{1}, z_{2}}(t)= \begin{cases}r & \text { if } I(t)=1 \text { and } X_{z_{2}}(t)<z_{1}, \\ v & \text { if } I(t)=1 \text { and } X_{z_{2}}(t)=z_{1}, \quad t \geq 0 \\ 0 & \text { if } I(t)=0\end{cases}
$$

The corresponding inventory process denoted by $X_{z_{1}, z_{2}}(t)$ can be described as follows:

(i) When $I(t)=1$ and $X_{z_{1}, z_{2}}(t)<z_{1}$ the process will increase with rate $r-v$ as time is going on.

(ii) When $I(t)=1$ and $X_{z_{1}, z_{2}}(t)=z_{1}$ the process will keep state $z_{1}$ until the system breaks down.

(iii) When $I(t)=0$ and $X_{z_{1}, z_{2}}(t)>-z_{2}$ the process will decrease with rate $-v$ as time is going on.

(iv) When $I(t)=0$ and $X_{z_{1}, z_{2}}(t)=-z_{2}$ the process will keep state $-z_{2}$ until the system starts over.

For convenience assume that at $t=0$ the system is in the up-state. A sample path of $X_{z_{1}, z_{2}}$ is given in Figure 1.

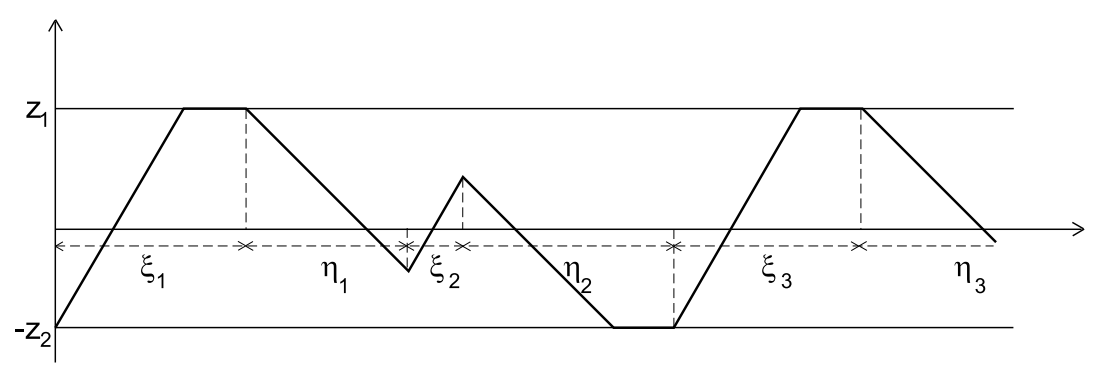

Fig. 1. Sample path of $X_{z_{1}, z_{2}}$

Briefly $X_{z_{1}, z_{2}}$ may be written in the following form:

$$
\begin{aligned}
& X_{z_{1}, z_{2}}(0)=y_{0} \in\left[-z_{2}, z_{1}\right], \\
& X_{z_{1}, z_{2}}(t)= \begin{cases}{\left[X_{z_{1}, z_{2}}\left(T_{2 n}\right)+(r-v)\left(t-T_{2 n}\right)\right] \wedge z_{1},} & T_{2 n}<t \leq T_{2 n+1}, \\
{\left[X_{z_{1}, z_{2}}\left(T_{2 n+1}\right)-v\left(t-T_{2 n+1}\right)\right] \vee\left(-z_{2}\right),} & T_{2 n+1}<t \leq T_{2 n+2},\end{cases}
\end{aligned}
$$

where

$T_{2 n}=\xi_{0}+\eta_{0}+\xi_{1}+\eta_{1}+\ldots+\xi_{n}+\eta_{n}, \quad T_{2 n+1}=T_{2 n}+\xi_{n+1}, \quad \xi_{0}=\eta_{0}=0$, and $n=0,1,2, \ldots$; we use the notation: $a \wedge b=\min (a, b), a \vee b=\max (a, b)$.

Observe that in the case $z_{2}=0$ the process is identical with that considered in [4]. For $z_{2}=\infty$ it coincides with that considered in [2]. 
(d) In this model, similarly to [2], the cost connected with the states of the process $X_{z_{1}, z_{2}}$ is given by the function $g: \mathbb{R} \rightarrow \mathbb{R}^{+}$such that

$$
g(x)= \begin{cases}c^{+} x & \text { if } x \geq 0, \\ -c^{-} x & \text { if } x<0 .\end{cases}
$$

where $c^{+}>0$ denotes the unit holding cost and $c^{-}>0$ the unit shortage (penalty) cost. Note that the cost function does not distinguish the state $-z_{2}$. But in this model this state differs from other shortage states. The parameter $z_{2}$ denotes the backlog limit. So if the process occupies the state $-z_{2}$ then the demand is lost. This fact should be stressed in the model. In the literature we find two methods: one is to add an additional penalty cost, the other is to add an additional constraint. We choose the second way. So in this model a constraint for the limiting probability of the state $-z_{2}$ is added.

The hedging parameters $z_{1}$ and $z_{2}$ are considered as decision variables. The problem is formulated as the following optimization problem.

\subsection{Optimization problem}

Problem 1. Let $\varepsilon \in[1,0)$. Find $z_{1} \geq 0, z_{2} \geq 0$ such that

$$
G\left(z_{1}, z_{2}\right)=\lim _{T \rightarrow \infty} \frac{1}{T} E \int_{0}^{T} g\left(X_{z_{1}, z_{2}}(t)\right) d t
$$

is minimal under the condition

$$
\lim _{t \rightarrow \infty} P\left\{X_{z_{1}, z_{2}}(t)=-z_{2}\right\}=\varepsilon .
$$

REMARK 2. It may happen that for some $\varepsilon$ the set of admissible parameters $z_{1}, z_{2}$ is empty. In that case Problem 1 does not make sense. In Section 4 we discuss condition (2) more precisely and rewrite the optimization problem in a different form (Problem 11).

In view of Remark 2 , in the next sections we use the following definition.

Definition 3. Problem 1 is well defined for $\varepsilon$ if

$$
\left\{z_{1} \geq 0, z_{2} \geq 0: \lim _{t \rightarrow \infty} P\left\{X_{z_{1}, z_{2}}(t)=-z_{2}\right\}=\varepsilon\right\} \neq \emptyset .
$$

3. Limit distribution of the inventory process. A sample path of the process $X_{z_{1}, z_{2}}$ is given in Figure 1. So it is clear that if we put $y_{0}=-z_{2}$ then

$$
X_{z_{1}, z_{2}}(t)+z_{2}=X_{z_{1}+z_{2}, 0}(t) \quad \text { with } \quad X_{z_{1}+z_{2}, 0}(0)=0 .
$$

This relation allows us to find the limit distribution of $X_{z_{1}, z_{2}}(t)$ provided we know the limit distribution of $X_{z_{1}+z_{2}, 0}$.

A sample path of $X_{z, 0}$ with $z=z_{1}+z_{2}$ is given in Figure 2 . 


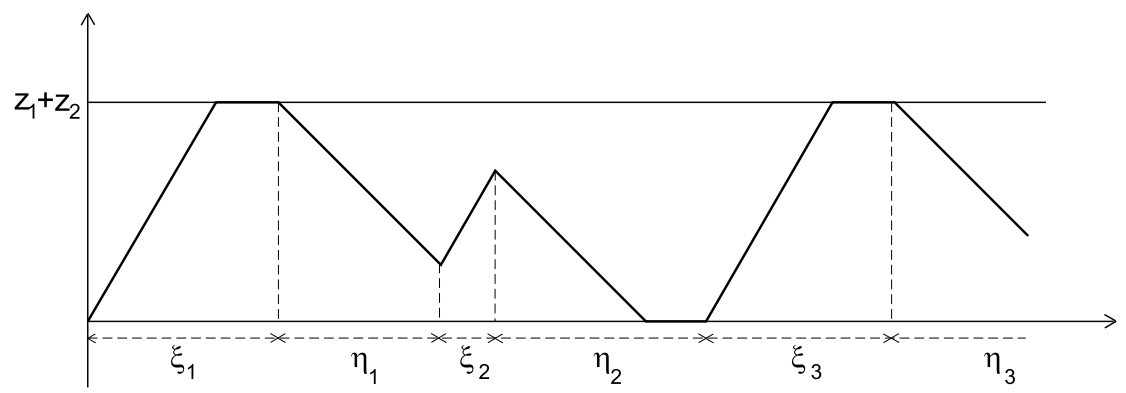

Fig. 2. Sample path of $X_{z_{1}+z_{2}, 0}$

$$
\begin{aligned}
& \text { Let } \\
& \begin{aligned}
P_{z_{1}, z_{2}}\left\{z_{1}\right\} & =\lim _{t \rightarrow \infty} P\left\{X_{z_{1}, z_{2}}(t)=z_{1}\right\}, \\
f_{z_{1}, z_{2}}(x) & =\lim _{\delta x \rightarrow 0} \frac{\lim _{t \rightarrow \infty} P\left\{x \leq X_{z_{1}, z_{2}}(t)<x+\delta x\right\}}{\delta x}, \quad-z_{2}<x<z_{1}, \\
P_{z_{1}, z_{2}}\left\{-z_{2}\right\} & =\lim _{t \rightarrow \infty} P\left\{X_{z_{1}, z_{2}}(t)=-z_{2}\right\} .
\end{aligned}
\end{aligned}
$$

By (2) we have the following result.

Proposition 4.

1. $P_{z_{1}, z_{2}}\left\{z_{1}\right\}=P_{z_{1}+z_{2}, 0}\left\{z_{1}+z_{2}\right\}$

2. $f_{z_{1}, z_{2}}(x)=f_{z_{1}+z_{2}, 0}\left(x+z_{2}\right), \quad-z_{2}<x<z_{1}$,

3. $P_{z_{1}, z_{2}}\left\{-z_{2}\right\}=P_{z_{1}+z_{2}, 0}\{0\}$.

The limiting distribution of the process $X_{z, 0}$ has been calculated by B. Liu and J. Cao [4]. Following their paper let

$$
\alpha=\frac{\lambda_{d}}{v}, \quad \beta=\frac{\lambda_{u}}{r-v}, \quad \gamma=\alpha-\beta
$$

and moreover let

$$
\begin{aligned}
& a_{1}=\frac{r}{v} \cdot \frac{\lambda_{d}}{\lambda_{u}+\lambda_{d}}, \quad a_{2}=\frac{\lambda_{d}}{\lambda_{u}+\lambda_{d}}, \quad a_{3}=1-a_{1}=1-\frac{r}{v} \cdot \frac{\lambda_{d}}{\lambda_{u}+\lambda_{d}}, \\
& a_{4}=a_{1}-a_{2}=\left(\frac{r}{v}-1\right) \frac{\lambda_{d}}{\lambda_{u}+\lambda_{d}} .
\end{aligned}
$$

Remark 5. Note that $\alpha>0, a_{1}>0, a_{2}>0$ and also $a_{4}>0, \beta>0$, because $r>v>0$.

The two lemmas below give some additional relations between the parameters.

LEMMA 6. $a_{3} \alpha+a_{4} \gamma=0$. 
Proof. We have

$$
\begin{aligned}
a_{3} \alpha+a_{4} \gamma & =\left(1-a_{1}\right) \alpha+\left(a_{1}-a_{2}\right)(\alpha-\beta) \\
& =\alpha-\alpha a_{1}+\alpha a_{1}-\alpha a_{2}-\beta a_{1}+\beta a_{2}=\left(1-a_{2}\right) \alpha+\left(a_{2}-a_{1}\right) \beta \\
& =\frac{\lambda_{u}}{\lambda_{u}+\lambda_{d}} \cdot \frac{\lambda_{d}}{v}-\left(\frac{r}{v}-1\right) \frac{\lambda_{d}}{\lambda_{u}+\lambda_{d}} \cdot \frac{\lambda_{u}}{r-v}=0 .
\end{aligned}
$$

LEMMA 7. If $\alpha=\beta$ then $a_{1}=1$ and $a_{3}=0$.

Proof. The assumption $\alpha=\lambda_{d} / v=\beta=\lambda_{u} /(r-v)$ means that $\lambda_{d} r=$ $\left(\lambda_{d}+\lambda_{u}\right) v$ and so $r / v=\left(\lambda_{u}+\lambda_{d}\right) / \lambda_{d}$. Hence $a_{1}=1$ and $a_{3}=0$.

By Theorem 3.2 of [4] and Lemma 7 we have the limit distribution for the process $X_{z, 0}$.

TheOREM 8 ([4, Theorem 3.2]).

1. If $\alpha=\beta$, then
(a) $f_{z, 0}(x)=\frac{\alpha}{1+\alpha z} \quad$ if $0<x<z$,
(b) $P_{z, 0}\{z\}=\frac{a_{2}}{1+\alpha z}$,
(c) $P_{z, 0}\{0\}=\frac{a_{4}}{1+\alpha z}$.

2. If $\alpha \neq \beta$, then
(a) $f_{z, 0}(x)=\frac{a_{1} \beta \gamma}{\alpha-\beta e^{-\gamma z}} e^{-\gamma(z-x)} \quad$ if $0<x<z$,
(b) $P_{z, 0}\{z\}=\frac{a_{2} \gamma}{\alpha-\beta e^{-\gamma z}}$,
(c) $P_{z, 0}\{0\}=a_{3}+\frac{a_{4} \gamma}{\alpha-\beta e^{-\gamma z}}$.

Theorem 8 together with (2) and Proposition 4 gives the limit distribution of the process $X_{z_{1}, z_{2}}(t)=X_{z_{1}+z_{2}, 0}(t)-z_{2}$.

Theorem 9. Put $s=z_{1}+z_{2}$.

1. If $\alpha=\beta$, then
(a) $P_{z_{1}, z_{2}}\left\{z_{1}\right\}=\frac{a_{2}}{1+\alpha s}$,
(b) $f_{z_{1}, z_{2}}(x)=\frac{\alpha}{1+\alpha s}$ if $-z_{2}<x<z_{1}$,
(c) $P_{z_{1}, z_{2}}\left\{-z_{2}\right\}=\frac{a_{4}}{1+\alpha s}$.

2. If $\alpha \neq \beta$, then
(a) $P_{z_{1}, z_{2}}\left\{z_{1}\right\}=\frac{a_{2} \gamma}{\alpha-\beta e^{-\gamma s}}$, 
(b) $f_{z_{1}, z_{2}}(x)=\frac{a_{1} \beta \gamma}{\alpha-\beta e^{-\gamma s}} e^{-\gamma\left(z_{1}-x\right)} \quad$ if $-z_{2}<x<z_{1}$,

(c) $P_{z_{1}, z_{2}}\left\{-z_{2}\right\}=a_{3}+\frac{a_{4} \gamma}{\alpha-\beta e^{-\gamma s}}$.

4. Condition (2) of Problem 1. Put as before $s=z_{1}+z_{2}$.

(i) Consider the case $\gamma=\alpha-\beta=0$ and put

$$
h_{\gamma}(s)=\frac{a_{4}}{1+a s} \text {. }
$$

By 1(c) of Theorem 9,

$$
\lim _{t \rightarrow \infty} P\left\{X_{z_{1}, z_{2}}(t)=-z_{2}\right\}=P_{z_{1}, z_{2}}\left\{-z_{2}\right\}=\frac{a_{4}}{1+a s}=h_{\gamma}(s) .
$$

In this case the definition of $a_{4}$ and Lemma 7 imply that

$$
a_{4}=a_{1}-a_{2}=1-\frac{\lambda_{d}}{\lambda_{u}+\lambda_{d}}=\frac{\lambda_{u}}{\lambda_{u}+\lambda_{d}}>0,
$$

which means that the function $h_{\gamma}(s)$ is strictly decreasing on $[0, \infty)$ with

$$
\max h_{\gamma}(s)=h_{\gamma}(0)=\frac{\lambda_{u}}{\lambda_{u}+\lambda_{d}}, \quad \inf h_{\gamma}(s)=\lim _{s \rightarrow \infty} h_{\gamma}(s)=0 .
$$

(ii) Consider the case $\gamma=\alpha-\beta \neq 0$. Now put

$$
h_{\gamma}(s)=a_{3}+\frac{a_{4} \gamma}{\alpha-\beta e^{-\gamma s}} .
$$

By Remark 5 we have $a_{4}>0$, hence $h_{\gamma}$ is strictly decreasing on $[0, \infty)$ because $h_{\gamma}^{\prime}(s)=-a_{4} \gamma^{2} \beta e^{-\gamma s} /\left(\alpha-\beta e^{-\gamma s}\right)^{2}<0$. So

$$
\max h_{\gamma}(s)=h_{\gamma}(0)=a_{3}+a_{4}=\frac{\lambda_{u}}{\lambda_{u}+\lambda_{d}} .
$$

(ii)' If $\gamma=\alpha-\beta>0$ then

$$
\inf h_{\gamma}(s)=\lim _{s \rightarrow \infty} h_{\gamma}(s)=a_{3}+a_{4} \frac{\gamma}{\alpha}=0
$$

by Lemma 6 .

(ii)" If $\gamma=\alpha-\beta<0$ then by (3) we have

$$
\begin{aligned}
\inf h_{\gamma}(s) & =\lim _{s \rightarrow \infty} h_{\gamma}(s)=a_{3}=1-\frac{r}{v} \cdot \frac{\lambda_{d}}{\lambda_{u}+\lambda_{d}}=\frac{v \lambda_{u}}{v\left(\lambda_{u}+\lambda_{d}\right)}+\frac{\lambda_{d}(v-r)}{v\left(\lambda_{u}+\lambda_{d}\right)} \\
& =\frac{\lambda_{u}}{\lambda_{u}+\lambda_{d}}-\frac{\lambda_{d}(r-v) \lambda_{u}}{v \lambda_{u}\left(\lambda_{u}+\lambda_{d}\right)}=\frac{\lambda_{u}}{\lambda_{u}+\lambda_{d}}\left(1-\frac{\alpha}{\beta}\right)>0 .
\end{aligned}
$$

Now we collect the results obtained. For brevity, we denote by $D_{\varepsilon}=$ $\left\{z_{1} \geq 0, z_{2} \geq 0: P_{z_{1}, z_{2}}\left\{-z_{2}\right\}=\varepsilon\right\}$ the set appearing in Definition 3 . 
Proposition 10.

1. If $\gamma=\alpha-\beta \geq 0$ then

(a) for $\varepsilon \in\left[1, \lambda_{u} /\left(\lambda_{u}+\lambda_{d}\right)\right)$ the set $D_{\varepsilon}$ is empty,

(b) for $\varepsilon \in\left[\lambda_{u} /\left(\lambda_{u}+\lambda_{d}\right), 0\right)$ there exists exactly one $s=h_{\gamma}^{-1}(\varepsilon)$ $\in[0, \infty)$ such that $D_{\varepsilon}=\left\{z_{1} \geq 0, z_{2} \geq 0: z_{1}+z_{2}=s\right\}$.

2. If $\gamma=\alpha-\beta<0$ then

(a) for

$$
\varepsilon \in\left[1, \frac{\lambda_{u}}{\lambda_{u}+\lambda_{d}}\right) \cup\left[\frac{\lambda_{u}}{\lambda_{u}+\lambda_{d}}-\frac{\alpha}{\beta} \cdot \frac{\lambda_{u}}{\lambda_{u}+\lambda_{d}}, 0\right)
$$

the set $D_{\varepsilon}$ is empty,

(b) for

$$
\varepsilon \in\left[\frac{\lambda_{u}}{\lambda_{u}+\lambda_{d}}, \frac{\lambda_{u}}{\lambda_{u}+\lambda_{d}}-\frac{\alpha}{\beta} \cdot \frac{\lambda_{u}}{\lambda_{u}+\lambda_{d}}\right)
$$

there exists exactly one $s=h_{\gamma}^{-1}(\varepsilon) \in[0, \infty)$ such that $D_{\varepsilon}=\left\{z_{1} \geq 0\right.$, $\left.z_{2} \geq 0: z_{1}+z_{2}=s\right\}$.

3. Problem 1 is well defined for $\varepsilon=h_{\gamma}(s)$ with $s \in[0, \infty)$. For all parameters $\gamma$ the function $h_{\gamma}(s)$ is strictly decreasing on $[0, \infty)$.

The case $\alpha<\beta$ needs some comment. The quantities $1 / \lambda_{d}$ and $1 / \lambda_{u}$ denote the mean down-time and the mean up-time of the system, respectively. So $1 / \alpha=\left(1 / \lambda_{d}\right) v$ is the total depletion in the mean down-time. Similarly $1 / \beta=\left(1 / \lambda_{u}\right)(r-v)$ is the total production in the mean up-time. Hence $\alpha<\beta$ implies that the total depletion in the mean down-time is greater then the total production in the mean up-time. This is the reason why the system cannot stay in the shortage state $-z_{2}$ with small probability as follows from the second part of $2(\mathrm{a})$.

4.1. Another formulation of the optimization problem. Proposition 10 allows us to consider the two hedging points optimization problem in the following form:

Problem 11. For given $s \geq 0$ find $z_{1}, z_{2}$ such that

$$
G_{s}\left(z_{1}, z_{2}\right)=\lim _{T \rightarrow \infty} \frac{1}{T} E \int_{0}^{T} g\left(X_{z_{1}, z_{2}}(t)\right) d t
$$

is minimal under the condition

$$
z_{1} \geq 0, z_{2} \geq 0, \quad z_{1}+z_{2}=s .
$$

For fixed hedging points $z_{1}, z_{2}$ the limit distribution of $X_{z_{1}, z_{2}}$ is given in Theorem 9. Using this distribution and the theory of regenerative processes ([1, Chap. V]) we have 


$$
G_{s}\left(z_{1}, z_{2}\right)=\int_{z_{2}}^{z_{1}} g(x) f_{z_{1}, z_{2}}(x) d x+c^{+} z_{1} P_{z_{1}, z_{2}}\left\{z_{1}\right\}+c^{-} z_{2} P_{z_{1}, z_{2}}\left\{-z_{2}\right\} .
$$

The subscript $s$ denotes that in Problem 11 the constant $s \in[0, \infty)$ is treated as a parameter.

Put

$$
\begin{array}{ll}
A=-c^{-} \int_{-z_{2}}^{0} x f_{z_{1}, z_{2}}(x) d x, & B=c^{+} \int_{0}^{z_{1}} x f_{z_{1}, z_{2}}(x) d x, \\
C=c^{+} z_{1} P_{z_{1}, z_{2}}\left\{z_{1}\right\}, & D=c^{-} z_{2} P_{z_{1}, z_{2}}\left\{-z_{2}\right\} .
\end{array}
$$

Then $G_{s}\left(z_{1}, z_{2}\right)=A+B+C+D$.

In Sections 5 and 6 we calculate $A, B, C, D$ and solve Problem 11 for the cases $\gamma=0, \gamma>0$ and $\gamma<0$.

5. Optimal solution for the case $\gamma=\alpha-\beta=0$. In this case $\alpha=$ $\lambda_{d} / v=\lambda_{u} /(r-v)=\beta$ and so (cf. Lemma 7) the constants (4) defined in Section 3 are

$$
a_{2}=\frac{v}{r}=\frac{\lambda_{d}}{\lambda_{u}+\lambda_{d}}, a_{1}=\frac{r}{v} a_{2}=1, a_{3}=0, a_{4}=a_{1}-a_{2}=1-\frac{v}{r} .
$$

By Theorem 9 putting $z_{2}=s-z_{1}$ we have

$$
\begin{aligned}
& A=-c^{-} \int_{-z_{2}}^{0} x \frac{\alpha}{1+\alpha s} d x=\frac{1}{2} c^{-} \frac{\alpha}{1+\alpha s}\left(s-z_{1}\right)^{2}, \\
& B=c^{+} \int_{0}^{z_{1}} x \frac{\alpha}{1+\alpha s} d x=\frac{1}{2} c^{+} \frac{\alpha}{1+\alpha s} z_{1}^{2}, \\
& C=c^{+} \frac{a_{2}}{1+\alpha s} z_{1}, \quad D=c^{-} \frac{a_{4}}{1+\alpha s}\left(s-z_{1}\right) .
\end{aligned}
$$

Hence

$$
G_{s}\left(z_{1}, s-z_{1}\right)=\frac{\alpha}{1+\alpha s}\left[\frac{c^{-}}{2}\left(s-z_{1}\right)^{2}+\frac{c^{+}}{2} z_{1}^{2}+\frac{a_{2} c^{+}}{\alpha} z_{1}+\frac{a_{4} c^{-}}{\alpha}\left(s-z_{1}\right)\right] .
$$

It is convenient to define two auxiliary functions.

(a) Put

$$
f\left(z_{1}\right)=\frac{c^{-}}{2}\left(s-z_{1}\right)^{2}+\frac{c^{+}}{2} z_{1}^{2}+\frac{a_{2} c^{+}}{\alpha} z_{1}+\frac{a_{4} c^{-}}{\alpha}\left(s-z_{1}\right) \quad \text { for } z_{1} \geq 0 .
$$

Note that $f\left(z_{1}\right)=G_{s}\left(z_{1}, s-z_{1}\right)(1+\alpha s) / \alpha$ for $z_{1} \in[0, s]$. Clearly,

$$
f^{\prime}\left(z_{1}\right)=c^{+} z_{1}+c^{-} z_{1}-c^{-} s+\frac{a_{2} c^{+}}{\alpha}-\frac{a_{4} c^{-}}{\alpha} \quad \text { for } z_{1} \geq 0 .
$$

(b) Put moreover

$$
F(s)=\frac{c^{-}}{c^{-}+c^{+}} s+\frac{1}{\alpha} \cdot \frac{a_{4} c^{-}-a_{2} c^{+}}{c^{-}+c^{+}} \quad \text { for } s \geq 0 .
$$


By (5),

(6) $F(s)=w_{1} s+w_{2} \quad$ with $\quad w_{1}=\frac{c^{-}}{c^{-}+c^{+}}, w_{2}=\frac{1}{\alpha r} \cdot \frac{(r-v) c^{-}-v c^{+}}{c^{-}+c^{+}}$.

It is easy to see that for $z_{1} \in[0, s]$,

$$
\begin{aligned}
& \text { if } z_{1}<F(s) \text { then } G_{s} \text { is decreasing, } \\
& \text { if } z_{1}>F(s) \text { then } G_{s} \text { is increasing. }
\end{aligned}
$$

Observe that $0<w_{1}<1$ and $\operatorname{sgn}\left(w_{2}\right)=\operatorname{sgn}\left((r-v) c^{-}-v c^{+}\right)$. Hence we consider two cases (cf. Figures 3 and 4 ).

(i) $c^{-} / c^{+}>v /(r-v)$. In this case there exists $s^{*}>0$ such that $F\left(s^{*}\right)=s^{*}$. So $s<F(s)$ for $s<s^{*}$ and $0<F(s) \leq s$ for $s \geq s^{*}$. Hence by (7) the solution of Problem 11 takes the form

$$
z_{1}^{*}=\left\{\begin{array}{ll}
s & \text { if } s<s^{*}, \\
F(s) & \text { if } s \geq s^{*},
\end{array} \quad z_{2}^{*}= \begin{cases}0 & \text { if } s<s^{*} \\
s-F(s) & \text { if } s \geq s^{*}\end{cases}\right.
$$

In brief, $z_{1}^{*}=\min (s, F(s))$ (cf. Figure 3 ) and $z_{2}^{*}=s-z_{1}^{*}$.

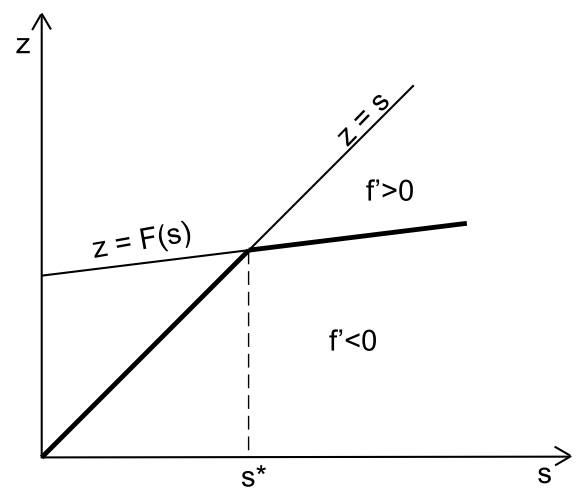

Fig. 3. $z_{1}^{*}=\min (s, F(s))$

(ii) $c^{-} / c^{+} \leq v /(r-v)$. This time let $s^{*}$ be such that $F\left(s^{*}\right)=0$. Then by (7) the solution of Problem 11 takes the form (cf. Figure 4)

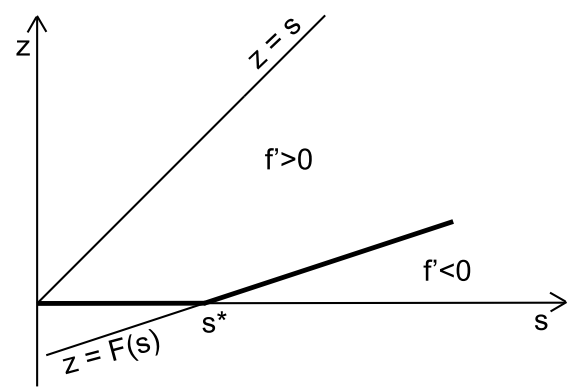

Fig. 4. $z_{1}^{*}=\max (0, F(s))$ 


$$
z_{1}^{*}=\left\{\begin{array}{ll}
0 & \text { if } s<s^{*}, \\
F(s) & \text { if } s \geq s^{*},
\end{array} \quad z_{2}^{*}= \begin{cases}s & \text { if } s<s^{*}, \\
s-F(s) & \text { if } s \geq s^{*}\end{cases}\right.
$$

In brief, $z_{1}^{*}=\max (0, F(s))$ and $z_{2}^{*}=s-z_{1}^{*}$.

Now we collect the results obtained.

5.0.1. Solution of Problem 11 for $\gamma=0$. Let $F(s)$ be given by (6) for $s \geq 0$. Suppose that $\alpha=\beta$.

(i) If $c^{-} / c^{+}>v /(r-v)$ then the optimal hedging points are: $z_{1}^{*}=$ $\min (s, F(s))$ and $z_{2}^{*}=s-z_{1}^{*}$ for $s \geq 0$.

(ii) If $c^{-} / c^{+} \leq v /(r-v)$ then the optimal hedging points are: $z_{1}^{*}=$ $\max (0, F(s))$ and $z_{2}^{*}=s-z_{1}^{*}$ for $s \geq 0$.

Remarks. Note that $\alpha=\beta$ implies that the total depletion in a mean down-time is equal to the maximum total production in a mean up-time. Recall that the inventory process decreases with rate $-v$ and increases with rate $r-v$ and $c^{-}$denotes the unit shortage cost and $c^{+}$the unit holding cost. Hence in the case $\alpha=\beta$ the optimal solution depends on the relation between the cost fraction $c^{-} / c^{+}$and the rate fraction $v /(r-v)$.

6. Optimal solution for the case $\gamma=\alpha-\beta \neq 0$. We recall that in this case $\alpha=\lambda_{d} / v \neq \lambda_{u} /(r-v)=\beta$, and so by (4),

$$
\begin{aligned}
& a_{1}=\frac{r}{v} a_{2} \neq 1, \quad a_{2}=\frac{\lambda_{d}}{\lambda_{u}+\lambda_{d}}, \quad a_{3}=1-a_{1}=1-\frac{r}{v} a_{2} \neq 0, \\
& a_{4}=a_{1}-a_{2}=\frac{r-v}{v} a_{2} .
\end{aligned}
$$

By Remark 5, we have $\alpha>0, \beta>0, a_{1}>0, a_{2}>0, a_{4}>0$. Lemma 6 states that

$$
a_{3} \alpha+a_{4} \gamma=0
$$

which implies that

$$
\operatorname{sgn}\left(a_{3}\right)=\operatorname{sgn}\left(1-a_{1}\right)=\operatorname{sgn}(-\gamma)
$$

By the second part of Theorem $9, G_{s}\left(z_{1}, z_{2}\right)=A+B+C+D$ where

$$
\begin{aligned}
A & =-\int_{-z_{2}}^{0} c^{-} x \frac{a_{1} \beta \gamma}{\alpha-\beta e^{-\gamma s}} e^{-\gamma\left(z_{1}-x\right)} d x=-c^{-} \frac{a_{1} \beta \gamma}{\alpha-\beta e^{-\gamma s}} e^{-\gamma z_{1}} \int_{-z_{2}}^{0} x e^{\gamma x} d x \\
& =-c^{-} \frac{a_{1} \beta \gamma}{\alpha-\beta e^{-\gamma s}} e^{-\gamma z_{1}}\left\{\left.\frac{e^{\gamma x}}{\gamma^{2}}(\gamma x-1)\right|_{-z_{2}} ^{0}\right\} \\
& =c^{-} \frac{a_{1} \beta \gamma}{\alpha-\beta e^{-\gamma s}} e^{-\gamma z_{1}}\left\{\frac{1}{\gamma^{2}}-\frac{e^{-\gamma z_{2}}}{\gamma^{2}} \gamma z_{2}-\frac{e^{-\gamma z_{2}}}{\gamma^{2}}\right\}
\end{aligned}
$$




$$
\begin{aligned}
B & =\int_{0}^{z_{1}} c^{+} x \frac{a_{1} \beta \gamma}{\alpha-\beta e^{-\gamma s}} e^{-\gamma\left(z_{1}-x\right)} d x=c^{+} \frac{a_{1} \beta \gamma}{\alpha-\beta e^{-\gamma s}} e^{-\gamma z_{1}} \int_{0}^{z_{1}} x e^{\gamma x} d x \\
& =c^{+} \frac{a_{1} \beta \gamma}{\alpha-\beta e^{-\gamma s}} e^{-\gamma z_{1}}\left\{\left.\frac{e^{\gamma x}}{\gamma^{2}}(\gamma x-1)\right|_{0} ^{z_{1}}\right\} \\
& =c^{+} \frac{a_{1} \beta \gamma}{\alpha-\beta e^{-\gamma s}} e^{-\gamma z_{1}}\left\{\frac{e^{\gamma z_{1}}}{\gamma^{2}} \gamma z_{1}-\frac{e^{\gamma z_{1}}}{\gamma^{2}}+\frac{1}{\gamma^{2}}\right\} ; \\
C & =c^{+} z_{1} \frac{a_{2} \gamma}{\alpha-\beta e^{-\gamma s}} ; \quad D=\left(a_{3}+\frac{a_{4} \gamma}{\alpha-\beta e^{-\gamma s}}\right) c^{-} z_{2} .
\end{aligned}
$$

Putting $z_{2}=s-z_{1}$ we have

$$
\begin{aligned}
A & =c^{-} \frac{a_{1} \beta \gamma}{\alpha-\beta e^{-\gamma s}} e^{-\gamma z_{1}}\left\{\frac{1}{\gamma^{2}}-\frac{e^{-\gamma s} e^{\gamma z_{1}}}{\gamma^{2}} \gamma s+\frac{e^{-\gamma s} e^{\gamma z_{1}}}{\gamma^{2}} \gamma z_{1}-\frac{e^{-\gamma s} e^{\gamma z_{1}}}{\gamma^{2}}\right\} \\
& =c^{-} \frac{a_{1} \beta \gamma}{\alpha-\beta e^{-\gamma s}}\left\{\frac{e^{-\gamma z_{1}}}{\gamma^{2}}-\frac{e^{-\gamma s}}{\gamma^{2}} \gamma s+\frac{e^{-\gamma s}}{\gamma^{2}} \gamma z_{1}-\frac{e^{-\gamma s}}{\gamma^{2}}\right\} ; \\
B & =c^{+} \frac{a_{1} \beta \gamma}{\alpha-\beta e^{-\gamma s}}\left\{\frac{\gamma z_{1}}{\gamma^{2}}-\frac{1}{\gamma^{2}}+\frac{e^{-\gamma z_{1}}}{\gamma^{2}}\right\} ; \\
C & \left.=c^{+} z_{1} \frac{v a_{1} \gamma}{r\left(\alpha-\beta e^{-\gamma s}\right)} \quad \text { (because } a_{2}=(v / r) a_{1}\right) .
\end{aligned}
$$

By Lemma $6, a_{3} \alpha+a_{4} \gamma=0$, hence we have

$$
\begin{aligned}
D & =\left(a_{3}+\frac{a_{4} \gamma}{\alpha-\beta e^{-\gamma s}}\right) c^{-}\left(s-z_{1}\right)=c^{-} \frac{a_{3} \alpha-a_{3} \beta e^{-\gamma s}+a_{4} \gamma}{\alpha-\beta e^{-\gamma s}}\left(s-z_{1}\right) \\
& =-c^{-} \frac{a_{3} \beta e^{-\gamma s}}{\alpha-\beta e^{-\gamma s}}\left(s-z_{1}\right) \\
& =\frac{a_{1} \gamma \beta}{\alpha-\beta e^{-\gamma s}}\left[-\frac{c^{-} a_{3}}{a_{1}} \cdot \frac{e^{-\gamma s}}{\gamma} s+\frac{c^{-} a_{3}}{a_{1}} \cdot \frac{e^{-\gamma s}}{\gamma} z_{1}\right] .
\end{aligned}
$$

Therefore

$$
\begin{aligned}
& G_{s}\left(z_{1}, s-z_{1}\right) \\
& =A+B+C+D \\
& =\frac{a_{1} \beta \gamma}{\alpha-\beta e^{-\gamma s}} \cdot \frac{1}{\gamma^{2}}\left[c^{-} e^{-\gamma z_{1}}-c^{-} e^{-\gamma s} \gamma s+c^{-} e^{-\gamma s} \gamma z_{1}-c^{-} e^{-\gamma s}\right. \\
& \left.\quad+c^{+} \gamma z_{1}-c^{+}+c^{+} e^{-\gamma z_{1}}+\frac{c^{+} \gamma^{2} v}{r \beta} z_{1}-\frac{c^{-} a_{3} \gamma}{a_{1}} e^{-\gamma s} s+\frac{c^{-} a_{3} \gamma}{a_{1}} e^{-\gamma s} z_{1}\right]
\end{aligned}
$$

We can show that the coefficient $\frac{a_{1} \beta \gamma}{\alpha-\beta e^{-\gamma s}} \cdot \frac{1}{\gamma^{2}}$ is strictly positive.

LEMMA 12. We have

$$
p(s)=\frac{a_{1} \beta \gamma}{\alpha-\beta e^{-\gamma s}} \cdot \frac{1}{\gamma^{2}}>0 \quad \text { for } s \geq 0 \text {. }
$$


Proof. Indeed,

$$
p^{\prime}(s)=-\frac{a_{1} \beta \gamma}{\left(\alpha-\beta e^{-\gamma s}\right)^{2}} \cdot \frac{\beta \gamma e^{-\gamma s}}{\gamma^{2}}=-\frac{a_{1} \beta^{2} e^{-\gamma s}}{\left(\alpha-\beta e^{-\gamma s}\right)^{2}}<0
$$

because $a_{1}>0$. Hence for $s \geq 0$ we have

$$
p(s)>\lim _{s \rightarrow \infty} p(s)= \begin{cases}\frac{a_{1} \beta}{\alpha \gamma}>0 & \text { if } \gamma>0, \\ 0 & \text { if } \gamma<0 .\end{cases}
$$

For the discussion of Problem 11 it is convenient to define for $z_{1} \geq 0$ an auxiliary function $\widetilde{f}\left(z_{1}\right)$ in the following way:

$$
\begin{aligned}
\widetilde{f}\left(z_{1}\right)= & \left(c^{-}+c^{+}\right) e^{-\gamma z_{1}}+\left(c^{-} e^{-\gamma s} \gamma+c^{+} \gamma+\frac{c^{+} \gamma^{2} v}{r \beta}+\frac{c^{-} a_{3} \gamma}{a_{1}} e^{-\gamma s}\right) z_{1} \\
& -\left(c^{-} \gamma s+c^{-}+\frac{c^{-} a_{3}}{a_{1}} \gamma s\right) e^{-\gamma s}-c^{+} .
\end{aligned}
$$

Then $\widetilde{f}\left(z_{1}\right)=G_{s}\left(z_{1}, s-z_{1}\right) / p(s)$ for $z_{1} \in[0, s]$. It is easy to see that

$$
\widetilde{f}^{\prime}\left(z_{1}\right)=-\gamma\left(c^{-}+c^{+}\right) e^{-\gamma z_{1}}+\gamma\left(c^{-} e^{-\gamma s}+c^{+}+\frac{c^{+} \gamma v}{r \beta}+\frac{c^{-} a_{3}}{a_{1}} e^{-\gamma s}\right) .
$$

Put

(10) $\widetilde{F}(s)=w_{1} e^{-\gamma s}+w_{2}$ where $w_{1}=\frac{c^{-}\left(1+\frac{a_{3}}{a_{1}}\right)}{c^{-}+c^{+}}, w_{2}=\frac{c^{+}\left(1+\frac{\gamma v}{r \beta}\right)}{c^{-}+c^{+}}$.

Then

$$
\begin{gathered}
\tilde{f}^{\prime}\left(z_{1}\right)=0 \quad \text { if and only if } e^{-\gamma z_{1}}=\widetilde{F}(s) ; \\
\text { if } \gamma>0 \text { then } \widetilde{f}^{\prime}\left(z_{1}\right) \geq 0 \text { if and only if } e^{-\gamma z_{1}} \leq \widetilde{F}(s) \text {; }
\end{gathered}
$$$$
\text { if } \gamma<0 \text { then } \widetilde{f}^{\prime}\left(z_{1}\right) \geq 0 \text { if and only if } e^{-\gamma z_{1}} \geq \widetilde{F}(s) \text {. }
$$

We discuss the coefficients $w_{1}, w_{2}$ more precisely.

First we show that

$$
w_{1}+w_{2}=1+\frac{1-a_{1}}{a_{1}\left(c^{-}+c^{+}\right)}\left(c^{-}-c^{+} \frac{\lambda_{d}}{\lambda_{u}}\right) .
$$

In fact by (10) we have

$$
\begin{aligned}
w_{1}+w_{2} & =1+\frac{1}{c^{-}+c^{+}}\left(c^{-} \frac{a_{3}}{a_{1}}+c^{+} \frac{\gamma v}{r \beta}\right) \\
& =1+\frac{1}{c^{-}+c^{+}} \frac{a_{3}}{a_{1}}\left(c^{-}+c^{+} \frac{\gamma v}{r \beta} \cdot \frac{a_{1}}{a_{3}}\right) .
\end{aligned}
$$


So by the definition of $\gamma, \alpha, \beta$ and the equality

$$
\frac{a_{1}}{a_{3}}=\frac{r \lambda_{d}}{(v-r) \lambda_{d}+v \lambda_{u}}
$$

one can get

$$
\begin{aligned}
c^{-}+c^{+} \frac{\gamma v}{r \beta} \cdot \frac{a_{1}}{a_{3}} & =c^{-}+c^{+} \frac{(\alpha-\beta) v}{r \beta} \cdot \frac{a_{1}}{a_{3}}=c^{-}+c^{+} \frac{v}{r} \cdot \frac{\frac{\lambda_{d}}{v}-\frac{\lambda_{u}}{r-v}}{\frac{\lambda_{u}}{r-v}} \cdot \frac{a_{1}}{a_{3}} \\
& =c^{-}+c^{+} \frac{v}{r} \cdot \frac{\frac{(v-r) \lambda_{d}+v \lambda_{u}}{v(v-r)}}{\frac{\lambda_{u}}{r-v}} \cdot \frac{a_{1}}{a_{3}} \cdot \frac{1}{c^{-}+c^{+}} \\
& =c^{-}+c^{+} \frac{v}{r} \cdot \frac{\left[(v-r) \lambda_{d}+v \lambda_{u}\right](r-v)}{v(v-r) \lambda_{u}} \cdot \frac{r \lambda_{d}}{(v-r) \lambda_{d}+v \lambda_{u}} \\
& =c^{-}-c^{+} \frac{\lambda_{d}}{\lambda_{u}} .
\end{aligned}
$$

Together with (15) and the equality $a_{3} / a_{1}=\left(1-a_{1}\right) / a_{1}$ this yields (14).

By a simple calculation (using the definitions of $a_{1}, \gamma, \alpha$ and $\beta$ ) one can see that

$$
\begin{aligned}
& w_{1}=\frac{c^{-}}{c^{-}+c^{+}} \cdot \frac{1}{a_{1}}=\frac{c^{-}}{c^{-}+c^{+}} \cdot \frac{v\left(\lambda_{u}+\lambda_{d}\right)}{r \lambda_{d}}>0, \\
& w_{2}=\frac{c^{+}\left(1+\frac{\gamma v}{r \beta}\right)}{c^{-}+c^{+}}=\frac{c^{+}}{c^{-}+c^{+}} \cdot \frac{(r-v)\left(\lambda_{u}+\lambda_{d}\right)}{r \lambda_{u}}>0 .
\end{aligned}
$$

6.1. The case $\gamma=\alpha-\beta>0$. In this case by (9) we have $a_{1}>1$. Hence by (16)-(17), $0<w_{1}<1$ and $w_{2}>0$. Note moreover that by (14),

$$
\widetilde{F}(0)=w_{1}+w_{2}=1+\frac{1-a_{1}}{a_{1}\left(c^{-}+c^{+}\right)}\left(c^{-}-c^{+} \frac{\lambda_{d}}{\lambda_{u}}\right)
$$

and so

(i) $0<\widetilde{F}(0)<1$ if $c^{-} / c^{+}>\lambda_{d} / \lambda_{u}$, and

(ii) $\widetilde{F}(0) \geq 1$ if $c^{-} / c^{+} \leq \lambda_{d} / \lambda_{u}$.

We discuss cases (i) and (ii) more precisely.

6.1.1. Solution of Problem 11 in case (i): $\gamma>0$ and $c^{-} / c^{+}>\lambda_{d} / \lambda_{u}$. Consider the problem of minimizing $G_{s}\left(z_{1}, s-z_{1}\right)$ for $z_{1} \in[0, s]$ with $s \geq 0$. The relation $z_{1} \in[0, s]$ means that

$$
1 \geq e^{-\gamma z_{1}} \geq e^{-\gamma s} .
$$


The inequalities $\widetilde{F}(0)<1$ and $\lim _{s \rightarrow \infty} \widetilde{F}(s)=w_{2}>0$ (cf. (17)) imply (cf. Figure 5) that there exists $s^{*}>0$ such that $\widetilde{F}\left(s^{*}\right)=e^{-\gamma s^{*}}$ and $\widetilde{F}(s)<e^{-\gamma s}$ for $s \in\left[0, s^{*}\right), \widetilde{F}(s) \geq e^{-\gamma s}$ for $s \in\left[s^{*}, \infty\right)$. Hence by $(12), \widetilde{f}^{\prime}\left(z_{1}\right) \geq 0$ if and only if $e^{-\gamma z_{1}} \leq \widetilde{F}(s)$. So by (18) the solution of Problem 11 is the following (cf. Figure 5):

$$
z_{1}^{*}=\left\{\begin{array}{ll}
s & \text { for } 0 \leq s \leq s^{*}, \\
-\frac{1}{\gamma} \ln \widetilde{F}(s) & \text { for } s>s^{*},
\end{array} z_{2}^{*}=s-z_{1}^{*} .\right.
$$

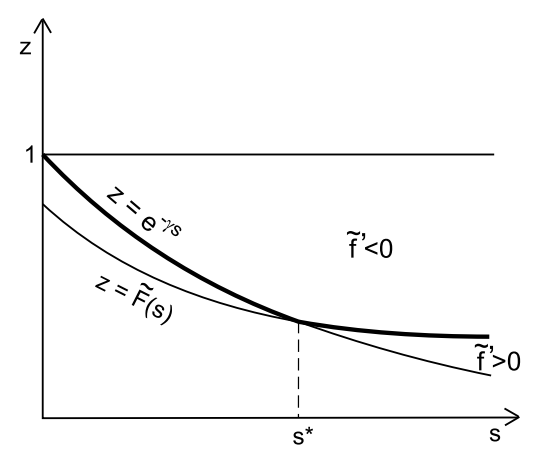

Fig. 5. $e^{-\gamma z_{1}^{*}}=\max \left\{e^{-\gamma s}, \widetilde{F}(s)\right\}$

6.1.2. Solution of Problem 11 in case (ii): $\gamma>0$ and $c^{-} / c^{+} \leq \lambda_{d} / \lambda_{u}$. As before, the relation $z_{1} \in[0, s]$ gives

$$
1 \geq e^{-\gamma z_{1}} \geq e^{-\gamma s} \text {. }
$$

The inequality $\widetilde{F}(s) \geq 1$ implies that there exists $s^{*} \in[0, \infty)$ such that $\widetilde{F}\left(s^{*}\right)=1$ provided $w_{2}<1$ (cf. Figure 6 ). By (12) in this case

$$
z_{1}^{*}=\left\{\begin{array}{ll}
0 & \text { for } 0 \leq s \leq s^{*}, \\
-\frac{1}{\gamma} \ln \widetilde{F}(s) & \text { for } s>s^{*},
\end{array} \quad z_{2}^{*}=s-z_{1}^{*} .\right.
$$

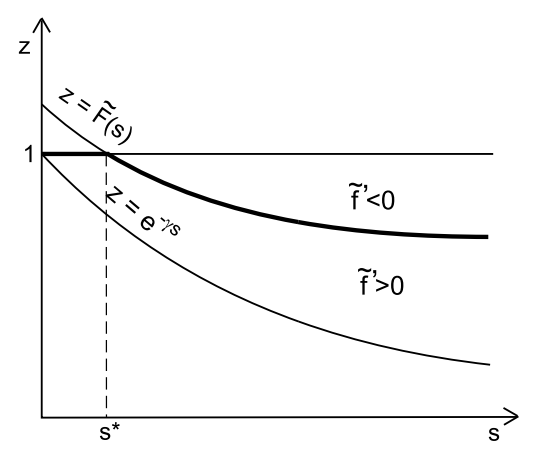

Fig. 6. $e^{-\gamma z_{1}^{*}}=\min \{1, \widetilde{F}(s)\}$ 
If $w_{2} \geq 1$ then $\widetilde{F}(s) \geq 1$ on $[0, \infty)$, hence by (12) we obtain

$$
z_{1}^{*}=0, \quad z_{2}^{*}=s .
$$

6.1.3. A special case with $\gamma>0$ and $s \rightarrow \infty$. Observe that if $s \rightarrow \infty$ (hence by Proposition 10, $\varepsilon \rightarrow 0$ ) then $\widetilde{F}(s) \rightarrow w_{2}$. Therefore (21) means that

$$
z_{1}^{*} \rightarrow 0 \quad \text { provided } w_{2} \geq 1 \text {. }
$$

Recall that

$$
w_{2}=\frac{c^{+}\left(1+\frac{\gamma v}{r \beta}\right)}{c^{-}+c^{+}} .
$$

So by (3),

$$
w_{2}=\frac{c^{+}}{c^{-}+c^{+}} \cdot \frac{(r-v)\left(\lambda_{u}+\lambda_{d}\right)}{r \lambda_{u}} .
$$

Hence (19) and (20) imply that if $w_{2}<1$ then

$$
z_{1}^{*} \rightarrow-\frac{1}{\gamma} \ln w_{2}=\frac{1}{\frac{\lambda_{d}}{v}-\frac{\lambda_{u}}{r-v}} \ln \frac{\left(c^{-}+c^{+}\right) r \lambda_{u}}{c^{+}(r-v)\left(\lambda_{u}+\lambda_{d}\right)} .
$$

In this special case we obtain formulas (5) and (7) from [2].

6.2. The case $\gamma=\alpha-\beta<0$. In this case by (9) we have $a_{1}<1$. Hence (16) and (17) imply that $w_{1}>0$ and $w_{2}>0$. Note that this time it may happen that $w_{1} \geq 1$. Note moreover that as before

$$
\widetilde{F}(0)=w_{1}+w_{2}=1+\frac{1-a_{1}}{a_{1}\left(c^{-}+c^{+}\right)}\left(c^{-}-c^{+} \frac{\lambda_{d}}{\lambda_{u}}\right)
$$

and so $a_{1}<1$ means that

(i) $)^{\prime} 0<\widetilde{F}(0)<1$ if $c^{-} / c^{+}<\lambda_{d} / \lambda_{u}$, and

(ii) $)^{\prime \prime} \widetilde{F}(0) \geq 1$ if $c^{-} / c^{+} \geq \lambda_{d} / \lambda_{u}$.

We discuss cases (i) ${ }^{\prime}$ and (ii)" more precisely.

6.2.1. Solution of Problem 11 in case (i) $)^{\prime}: \gamma<0$ and $c^{-} / c^{+}<\lambda_{d} / \lambda_{u}$. Observe that in this case there exists $s^{*}>0$ such that $\widetilde{F}\left(s^{*}\right)=1$ and $\widetilde{F}(s)<1$ for $s<s^{*}, \widetilde{F}(s) \geq 1$ for $s \geq s^{*}$. Moreover $\widetilde{F}(s)=w_{1} e^{-\gamma s}+w_{2}<e^{-\gamma s}$ because $\widetilde{F}(0)=w_{1}+w_{2}<1$ and so $w_{1}<1$. Hence (13) and the constraint $1 \leq e^{-\gamma z_{1}} \leq e^{-\gamma s}$ imply that the optimal solution is of the following form (cf. Figure 7):

$$
z_{1}^{*}=\left\{\begin{array}{ll}
0 & \text { for } 0 \leq s \leq s^{*}, \\
-\frac{1}{\gamma} \ln \widetilde{F}(s) & \text { for } s>s^{*},
\end{array} z_{2}^{*}=s-z_{1}^{*} .\right.
$$




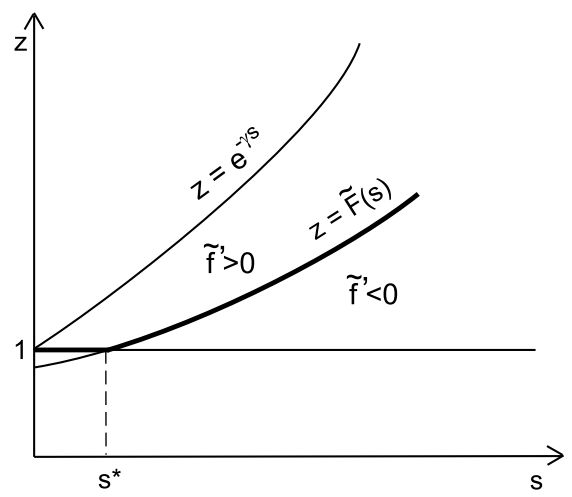

Fig. 7. $e^{-\gamma z_{1}^{*}}=\max \{1, \widetilde{F}(s)\}$

6.2.2. Solution of Problem 11 in case (ii) $)^{\prime} \gamma<0$ and $c^{-} / c^{+} \geq \lambda_{d} / \lambda_{u}$. In this case either $\widetilde{F}(s) \geq e^{-\gamma s}$, and hence by (13),

$$
z_{1}^{*}=s, \quad z_{2}^{*}=0,
$$

or there exists $s^{*} \geq 0$ such that $\widetilde{F}\left(s^{*}\right)=e^{-\gamma s^{*}}, \widetilde{F}(s) \geq e^{-\gamma s}$ on $\left[0, s^{*}\right]$ and $\widetilde{F}(s)<e^{-\gamma s}$ on $\left(s^{*}, \infty\right)$, hence (cf. Figure 8) by (13),

$$
z_{1}^{*}=\left\{\begin{array}{ll}
s & \text { for } 0 \leq s \leq s^{*}, \\
-\frac{1}{\gamma} \ln \widetilde{F}(s) & \text { for } s>s^{*},
\end{array} \quad z_{2}^{*}=s-z_{1}^{*} .\right.
$$

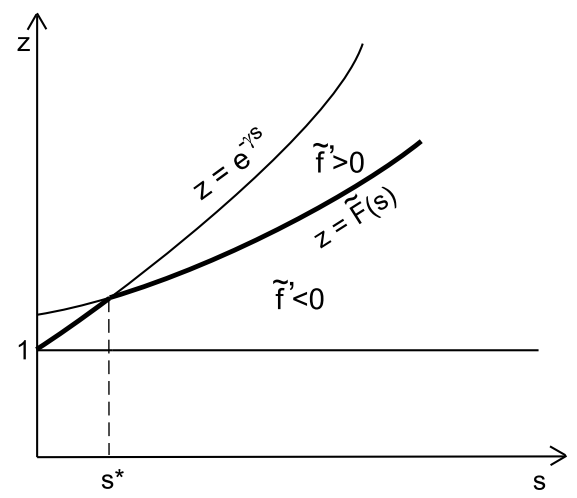

Fig. 8. $e^{-\gamma z_{1}^{*}}=\min \left\{e^{-\gamma s}, \widetilde{F}(s)\right\}$

Note that the last formula is true if there exists $s^{*} \geq 0$ such that $\widetilde{F}\left(s^{*}\right)=$ $w_{1} e^{-\gamma s^{*}}+w_{2}=e^{-\gamma s^{*}}$, which is true if $w_{2} /\left(1-w_{1}\right) \geq 1$.

7. Final remarks. We have obtained an optimal two hedging points policy for a version of the unreliable manufacturing system discussed in [2] 
and [4]. In the proof we essentially use the limit distribution obtained in [4]. In the special case $\gamma>0$ and $s \rightarrow \infty$ the solution of the problem reduces to formulas (5) and (7) of [2].

We should remark that another direction of development of the problem investigated in [2] for multiproduct models is presented in [3] and the references therein.

\section{References}

[1] S. Asmussen, Applied Probability and Queues, Wiley, 1987.

[2] T. Bielecki and P. R. Kumar, Optimality of zero-inventory policies for unreliable manufacturing systems, Oper. Res. 36 (1987), 532-541.

[3] T. E. Duncan, B. Pasik-Duncan and Ł. Stettner, Average cost per unit time control of stochastic manufacturing systems: revisited, Math. Methods Oper. Res. 54 (2001), 259-278.

[4] B. Liu and J. Cao, Production control of an unreliable manufacturing system under the assumption of no backlog, Math. Meth. Oper. Res. 46 (1997), 103-117.

[5] D. C. Montgomery, M. S. Bazaraa and A. K. Keswani, Inventory models with a mixture of backorders and lost sales, Naval Res. Logist. 20 (1973), 255-263.

[6] D. Rosenberg, A new analysis of a lot size model with partial backordering, ibid. 26 (1979), 349-353.

[7] H. M. Wee, Deteriorating inventory model with quantity discount, pricing and partial backordering, Internat. J. Production Econom. 59 (1999), 511-518.

Institute of Mathematics

Polish Academy of Sciences

Śniadeckich 8

00-950 Warszawa, Poland

E-mail: ryszrem@impan.gov.pl

Received on 12.10.2001;

revised version on 13.6.2002 